\title{
The Goddard expansion and the kinetic theory for solutions of rodlike macromolecules
}

\author{
S. I. Abdel-Khalik, Ole Hassager, and R. Byron Bird \\ Rheology Research Center and Chemical Engineering Department, University of Wisconsin, Madison, Wisconsin 53706 \\ (Received 30 April 1974)

\begin{abstract}
The Goddard memory-integral expansion for the stress tensor of viscoelastic fluids differs from previous expansions in that it uses a deformation rate referred to a co-rotating reference frame. It is shown how to get the kernel functions in the Goddard series in terms of the structural parameters occurring in the kinetic theory of macromolecular solutions. That the series has much better convergence properties than previously used expansions is demonstrated by comparison with exact kinetic theory results for steady-state shear flow and elongational flow.
\end{abstract}

\section{INTRODÚCTION}

In the earliest researches on the kinetic theory of macromolecules, investigators had the limited objective of obtaining the stress components for specialized simple flows, such as steady state shear flow or small-amplitude oscillatory motion. Then in 1956 Lodge l succeeded $^{1}$ in obtaining the complete expression for the stress tensor in terms of the kinematic history (i.e., the "rheological equation of state" or "constitutive equation") for a network model describing concentrated polymer solutions or melts. At about the same time Giesekus ${ }^{2}$ and Prager ${ }^{3}$ made the pioneering attempts to obtain the constitutive equation for a dilute solution of rigid macromolecules, using the rigid-dumbbell model. They considered only homogeneous flows which are slowly varying in time and obtained the coefficients of the first few terms in the "retarded-motion expansion" for the stress tensor in terms of the structural parameters of the model. Then more recently it was shown ${ }^{4}$ how to express the rigid-dumbbell kinetic-theory results in terms of an Oldroyd model. In addition Armstrong and $\mathrm{Bird}^{5,6}$ showed how to obtain kinetic theory expressions for the first few kernel functions of the covariant convected memory-integral expansion developed by Green, Rivlin, and Spencer, ${ }^{7,8}$ Coleman and Noll, ${ }^{9}$ and Pipkin ${ }^{10}$ (we shall refer to this as the GRSCNP expansion). In this paper we show that the Goddard ${ }^{11}$ memory-integral expansion is potentially more useful for presenting kinetic theory results.

We consider the flow of an incompressible fluid with velocity field $\mathbf{v}(\mathbf{r}, t)$. We use the following kinematic tensors:

$$
\begin{aligned}
& \dot{\gamma}=\nabla v+(\nabla v)^{\dagger}=\text { rate of deformation tensor } \\
& \omega=\nabla v-(\nabla v)^{\dagger}=\text { vorticity tensor } \\
& w=\frac{1}{2}[\nabla \times v]=\text { angular velocity vector } .
\end{aligned}
$$

Note that $\omega_{j k}=2 \sum_{i} \epsilon_{j k i} w_{i}$, that $(\nabla \mathbf{v})_{i j}=\left(\partial / \partial x_{i}\right) v_{j}$, and that $\dagger$ denotes the transpose.

Kinetic theory equations have customarily been formulated and solved for an observer translating (but not rotating) with a fluid particle. Here, however, we wish to formulate the equations for an observer translating with a fluid particle and rotating with angular velocity equal to the local fluid angular velocity vector $w=\frac{1}{2}[\nabla$ $\mathbf{x} \mathbf{v}$. Provided both observers solve their kinetic equations completely, they will of course obtain equivalent answers. If, however, they perform perturbation solutions we shall see that their respective expansions have very different convergence properties. Let us endow both the translating and the rotating observer with a set of mutually orthogonal unit vectors $\left(\delta_{1}, \delta_{2}, \delta_{3}\right.$ and $\check{\delta}_{1}$, $\check{\delta}_{2}, \check{\delta}_{3}$, respectively) which they use as basis for resolving vectors and tensors into their components. The two sets of vectors coincide at the present time $t$, and are at other times $t^{\prime}$ related by the orthogonal matrix function $Q_{i j}=Q_{i j}\left(t, t^{\prime}\right)$ as follows:

$$
\check{\delta}_{i}\left(t^{\prime}\right)=\sum_{j} Q_{i j} \delta_{j} .
$$

(Note: Goddard and Miller ${ }^{12}$ used a rotation tensor $Q_{t}^{t}$, related to our matrix elements by $\left.Q_{t}^{t}=\sum_{i} \sum_{j} Q_{f i} \delta_{i} \delta_{j}.\right)$

The time rate of change of $\check{\delta}_{i}\left(t^{\prime}\right)$ following a fluid particle is

$$
\frac{D}{D t^{\prime}} \check{\delta}_{i}=\left[\mathrm{w}\left(t^{\prime}\right) \times \check{\delta}_{i}\right]=-\frac{1}{2}\left[\omega\left(t^{\prime}\right) \cdot \check{\delta}_{i}\right] .
$$

Then from Eqs. (4) and (5) we obtain the following differential equation for the $Q_{i j}$ :

$$
\frac{D}{D t^{\prime}} Q_{i j}=\frac{1}{2} \sum_{m} Q_{i m} \omega_{m j}\left(t^{\prime}\right)
$$

with $Q_{i j}=\delta_{i j}$ at $t^{\prime}=t$.

By standard rules for transformations under a change of reference frame ${ }^{13,14}$ it follows that the components of the stress tensor, the rate of deformation tensor, and the vorticity tensor as seen by the rotating observer (that is, $\check{\tau}_{i j}, \check{\gamma}_{i j}, \check{\omega}_{i j}$ ) are related to those seen by the nonrotating observer $\left(\tau_{i j}, \dot{\gamma}_{i j}, \omega_{i j}\right)$ as follows:

$$
\begin{aligned}
& \check{\tau}_{i j}=\sum_{m} \sum_{n} Q_{i m} \tau_{m n} Q_{j n}, \\
& \check{\gamma}_{i j}=\sum_{m} \sum_{n} Q_{i m} \dot{\gamma}_{m n} Q_{j n} \equiv \dot{\Gamma}_{i j}\left(t, t^{\prime}\right), \\
& \check{\omega}_{i j}=\sum_{m} Q_{i m}\left(\sum_{n} \omega_{m n} Q_{j n}+2 \frac{D}{D t^{\prime}} Q_{j m}\right)=0 .
\end{aligned}
$$

The fact that $\tilde{\omega}_{i j}=0$ follows directly from Eq. (6); hence to the rotating observer the entire flow history appears to be irrotational. If the rotating observer performs a 
solution of his kinetic theory equations to obtain $\dot{\boldsymbol{\tau}}_{i j}(t)$ in terms of the history of $\check{\gamma}_{i j}\left(t^{\prime}\right)$ (with $-\infty<t^{\prime} \leq t$ ), this solution may be expressed in the nonrotating frame simply by replacing $\check{\tau}_{i j}(t)$ by $\tau_{i j}(t)$, and $\check{\gamma}_{i j}\left(t^{\prime}\right)$ by $\dot{\Gamma}_{i j}\left(t, t^{\prime}\right)$. This procedure has the advantage that the resulting equation automatically will be a properly formulated ${ }^{15}$ constitutive equation, whereas the fixed observer may have to recast his equation somewhat in order for his result to have a form which clearly satisfies all invariance requirements. This is particularly true when a perturbation expansion is performed; for example see the rearrangement performed in going from Eq. (22) to Eqs. (3), (23)-(26) in Ref. 6.

\section{KINETIC THEORY RESULTS FOR DILUTE SOLUTIONS OF RIGID, RODLIKE MACROMOLECULES}

For the purpose of studying the rheological behavior of rigid rodlike macromolecular solutions, we idealize the macromolecule as a rod of length $L$ with $N$ beads uniformly distributed over its length. Each bead has a friction coefficient $\zeta$ and there are $n_{0}$ macromolecules per unit volume. Hydrodynamic interaction, bending of the macromolecule, and excluded volume effects are neglected. The solution is presumed to be very dilute so that macromolecule-macromolecule interactions may be neglected, and the solvent is Newtonian and incompressible, with viscosity $\eta_{s}$. The structural parameters $\zeta$ and $L$ will always appear in the grouping

$$
\lambda=\frac{\zeta L^{2} N(N+1)}{72(N-1) k T}
$$

which is a time constant for the macromolecular solution.

It was shown by Armstrong and Bird ${ }^{6}$ how the kinetic theory equations for the above model can be solved for arbitrary time-dependent flows by means of a successive-approximation process involving perturbation around the equilibrium state. Now the entire development of Armstrong and Bird can be repeated with the observer in a coordinate frame rotating with the local angular velocity of the fluid. As mentioned in the previous section it is then necessary to solve the kinetictheory equations only for time-dependent, irrotational flows. This means then that Eq. (22) of Ref. 6 can be taken over directly by reinterpreting all tensor components as being referred to a local co-rotating frame. Of course, in adopting this interpretation, steady-state shear flow $v_{x}=v_{x}(y)$ as viewed by an observer fixed in space corresponds to a two-dimensional, large-amplitude, oscillatory irrotational flow as viewed by an observer going with the fluid and rotating with it.

When the Armstrong-Bird stress tensor expression, Eq. (22) of Ref. 6, is taken over in this way we obtain:

$$
\begin{aligned}
\boldsymbol{T}=\boldsymbol{\tau}_{s}-n_{0} k T & \lambda\left[\frac{2}{5} \dot{\Gamma}+\frac{3}{5 \lambda} \int_{-\infty}^{t} \exp \left(-\frac{t-t^{\prime}}{\lambda}\right) \dot{\Gamma}^{\prime} d t^{\prime}\right. \\
& +\frac{6}{35} \int_{-\infty}^{t} \exp \left(-\frac{t-t^{\prime}}{\lambda}\right)\left(\dot{\Gamma} \cdot \dot{\Gamma}^{\prime}+\dot{\Gamma}^{\prime} \cdot \dot{\Gamma}\right) d t^{\prime} \\
& +\frac{9}{70 \lambda} \int_{-\infty}^{t} \int_{-\infty}^{t^{\circ}} \exp \left(-\frac{t-t^{\prime \prime}}{\lambda}\right)
\end{aligned}
$$

$$
\left.\times\left(\dot{\Gamma}^{\prime} \cdot \dot{\Gamma}^{\prime \prime}+\dot{\Gamma}^{\prime \prime} \cdot \dot{\Gamma}^{\prime}\right) d t^{\prime \prime} d t^{\prime}+\cdots\right]
$$

in which $\tau_{s}$ is the solvent contribution to the stress tensor, $\dot{\Gamma}_{i j} \equiv \dot{\Gamma}_{i j}(t, t)=\dot{\gamma}_{i j}(t)$, and $\dot{\Gamma}_{i j}^{\prime} \equiv \dot{\Gamma}_{i j}\left(t, t^{\prime}\right)$ are defined in Eq. (8). The complete third-order term can be written down by analogy with Eq. (22) of Ref. 6; in that equation, however, the erroneous coefficient $27 / 280$ must be replaced by the correct value $27 / 980$. Equation (11), including the third-order terms, and Eq. (29) of Ref. 6 give identical results up through terms cubic in the rate of deformation.

\section{THE GODDARD EXPANSION}

By using techniques similar to those employed by previous investigators, ${ }^{7-10}$ Goddard ${ }^{11}$ showed that for an incompressible, isotropic viscoelastic fluid the stress tensor can be expanded in a memory-integral expansion using $\stackrel{\dot{\Gamma}}{\Gamma}$ as a kinematic tensor. This expansion, through third order, is

$$
\begin{aligned}
\boldsymbol{\tau}= & -\int_{-\infty}^{t} G_{\mathrm{I}}\left(t-t^{\prime}\right) \dot{\Gamma}^{\prime} d t^{\prime} \\
& -\frac{1}{2} \int_{-\infty}^{t} \int_{-\infty}^{t} G_{\mathrm{II}}\left(t-t^{\prime}, t-t^{\prime \prime}\right)\left[\dot{\Gamma}^{\prime} \cdot \dot{\Gamma}^{\prime \prime}+\dot{\Gamma}^{\prime \prime} \cdot \dot{\Gamma}^{\prime}\right] d t^{\prime \prime} d t^{\prime} \\
& -\frac{1}{2} \int_{-\infty}^{t} \int_{-\infty}^{t} \int_{-\infty}^{t}\left\{2 G_{\mathrm{III}}\left(t-t^{\prime}, t-t^{\prime \prime}, t-t^{\prime \prime \prime}\right) \dot{\Gamma}^{\prime} \dot{\Gamma}^{\prime \prime}: \dot{\Gamma}^{\prime \prime \prime}\right. \\
& \left.+G_{\mathrm{IV}}\left(t-t^{\prime}, t-t^{\prime \prime}, t-t^{\prime \prime \prime}\right)\left[\dot{\Gamma}^{\prime} \cdot \dot{\Gamma}^{\prime \prime} \cdot \dot{\circ}^{\prime \prime \prime}+\dot{\Gamma}^{\prime \prime \prime} \cdot \dot{\Gamma}^{\prime \prime} \cdot \dot{\Gamma}^{\prime}\right]\right\} \\
& \times d t^{\prime \prime \prime} d t^{\prime \prime} d t^{\prime}+\cdots .
\end{aligned}
$$

Here $G_{I}\left(t-t^{\prime}\right)$ is identical to the "relaxation modulus" used in linear viscoelasticity. By comparing Eqs. (11) and (12) it is possible to obtain the expressions for the kernel functions in terms of the structural parameters of the rigid bead-rod model considered in Sec. II:

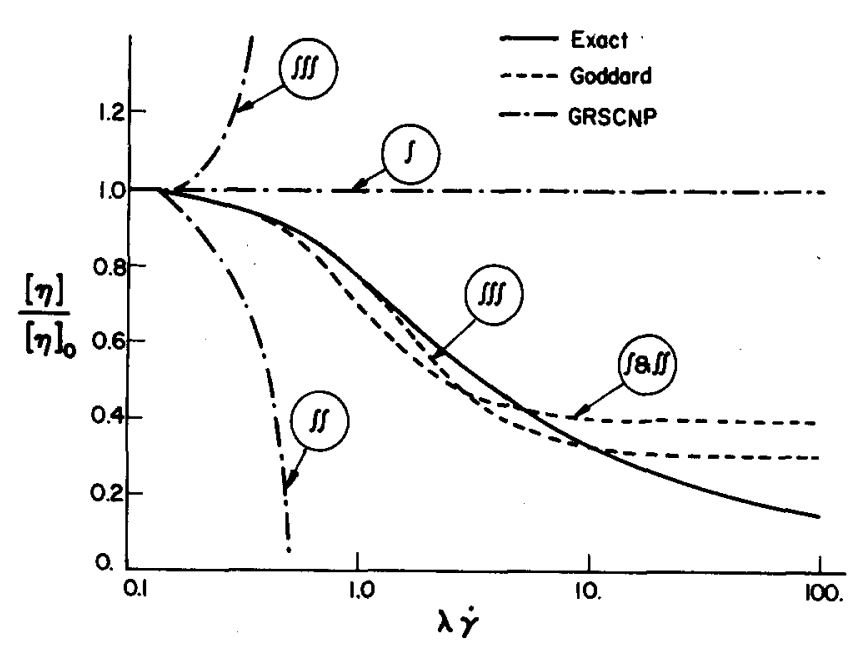

FIG. 1. Comparison of the exact intrinsic viscosity (as computed by Stewart and S6rensen ${ }^{17}$ ) for solutions of rigid rodlike macromolecules with the results obtained using a finite number of terms of the Goddard and the GRSCNP expansions. The number of integrals indicates the number of terms used in the expansions. 


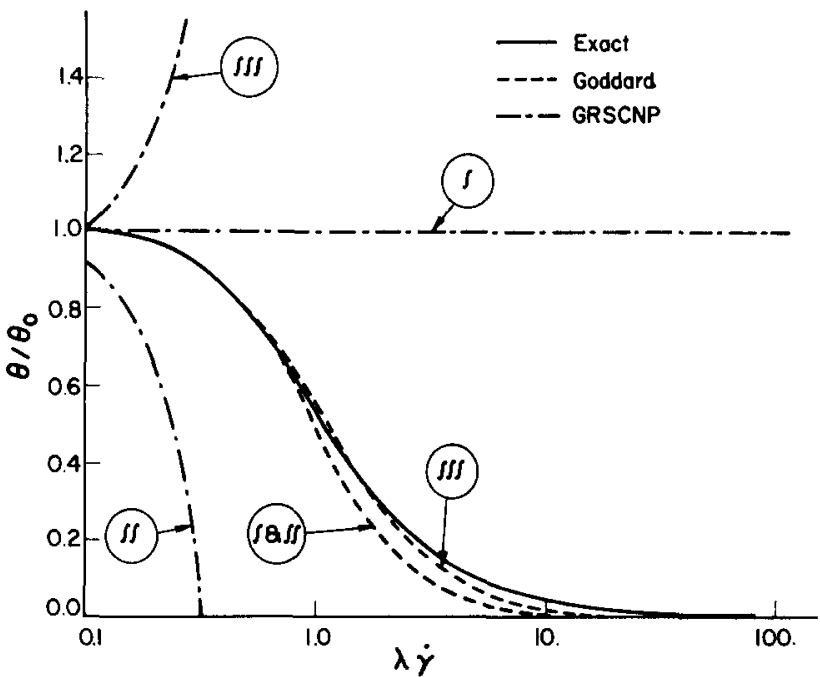

FIG. 2. Comparison similar to Fig. 1, for primary normal stress function $\theta(\dot{\gamma})$.

$$
\begin{aligned}
G_{I}\left(t-t^{\prime}\right)= & 2 \eta_{s} \delta\left(t-t^{\prime}\right) \\
+ & \frac{n_{0} k T \lambda}{5}\left[4 \delta\left(t-t^{\prime}\right)+\frac{3}{\lambda} \exp \left(-\frac{t-t^{\prime}}{\lambda}\right)\right] \\
G_{I I}\left(t-t^{\prime}, t-t^{\prime \prime}\right) & =\frac{n_{0} k T \lambda}{70}\left[24 \delta\left(t-t^{\prime}\right)\right. \\
& \left.+\frac{9}{\lambda} g\left(t^{\prime}, t^{\prime \prime}\right)\right] \exp \left(-\frac{t-t^{\prime \prime}}{\lambda}\right)
\end{aligned}
$$

in which

$$
g\left(t^{\prime}, t^{\prime \prime}\right)= \begin{cases}1 & \text { for }-\infty<t^{\prime \prime} \leqslant t^{\prime} \\ 0 & \text { for } t^{\prime}<t^{\prime \prime} \leqslant t\end{cases}
$$

and the $\delta(s)$ is defined such that:

$$
\int_{0}^{\infty} \delta(s) d s=\frac{1}{2} \text { and } \int_{-\infty}^{+\infty} \delta(s) d s=1 .
$$

Hence the rigid-rod, multibead model can be put into the form of the Goddard expansion.

The first term of the Goddard series with Eq. (13) for $G_{\text {I }}$ reproduces exactly all the linear viscoelastic kinetic theory results for rigid macromolecular solutions, including for example, $\eta^{\prime}$ and $\eta^{\prime \prime}$. It also gives the complete results for the oscillatory primary normal stresses (i.e., the amplitude functions $\theta^{\prime}$ and $\theta^{\prime \prime}$, and the displacement function $\theta^{d}$ ). Use of the first two terms in the Goddard series with Eqs. (13) and (14) for $G_{I}$ and $G_{I I}$ reproduces exactly the results for the oscillatory second-

TABLE I. The material functions for steady shear flow of solutions of rigid macromolecules as obtained from the Goddard expansion $\left[x=(\lambda \dot{\gamma})^{2}\right]$.

\begin{tabular}{llll}
\hline $\begin{array}{l}\text { Contribution } \\
\text { due to term } \\
\text { of order: }\end{array}$ & $\frac{\eta-\tau_{i s}}{n_{0} k T \lambda}$ & $\frac{\theta}{\frac{6}{5} n_{0} k T \lambda^{2}}$ & $\frac{\beta}{\frac{6}{5} n_{0} k T \lambda^{2}}$ \\
\hline 1 & $\frac{2}{5}+\frac{3}{5} \frac{1}{1+x}$ & $\frac{1}{1+x}$ & $-\frac{1}{2} \frac{1}{1+x}$ \\
2 & 0 & 0 & $\frac{1}{2} \frac{1}{1+x}$ \\
3 & $\frac{375 x+1335 x^{2}-144 x^{3}}{175(1+x)^{2}(25+9 x)}$ & $\frac{-3 x+293 x^{2}}{35(1+x)^{2}(25+9 x)}$ & $\frac{3 x-293 x^{2}}{70(1+x)^{2}(25+9 x)}$ \\
\hline
\end{tabular}

TABLE II. The material functions for steady shear flow of solutions of rigid macromolecules as obtained from the GRSCNP expansion $\left[x=(\lambda \dot{\gamma})^{2}\right]$.

\begin{tabular}{llcc}
\hline \hline $\begin{array}{l}\text { Contribution } \\
\text { due to term of } \\
\text { order: }\end{array}$ & $\frac{\eta-\eta_{s}}{n_{0} k T \lambda}$ & $\frac{\theta}{\frac{6}{5} n_{0} k T \lambda^{2}}$ & $\frac{\beta}{\frac{6}{5} n_{0} k T \lambda^{2}}$ \\
\hline 1 & 1 & 1 & -1 \\
2 & $-\frac{102}{35} x$ & $-\frac{60}{7} x$ & $1+\frac{60}{7} x$ \\
3 & $\frac{12}{5} x+\frac{253452}{6125} x^{2}$ & $\frac{262}{35} x+\frac{175748}{875} x^{2}$ & $-\frac{532}{35} x-\frac{175748}{875} x^{2}$ \\
\hline
\end{tabular}

ary normal stresses (i.e., the amplitude functions $\beta^{\prime}$ and $\beta^{\prime \prime}$, and the displacement function $\beta^{d}$ ). (See Ref. 16 , Sec. 7 for a summary of kinetic theory results for the oscillatory experiments.)

\section{COMPARISON OF THE GODDARD AND GRSCNP EXPANSIONS}

Once kinetic theory results are available they can be used to evaluate the constants or functions which appear in the various available constitutive equations. However, some constitutive relations may be more useful than others in that they can be used to extend the results at least approximately to higher rates of deformation. In this section we compare the Goddard expansion of Sec. III [or its equivalent in Eq. (11)] with the GRSCNP expansion used previously by Armstrong and Bird. ${ }^{6}$ It will be seen that there is an immense difference in the behavior of these two expansions. This was to be expected in view of Goddard's comment that his model for steady viscometric flows "gives higher order terms for the material functions, each of which is composed of a power of $\dot{\gamma}$ multiplied by a Fourier integral of a hereditary function. On the other hand, the expansions of Coleman and Noll, or of Green and Rivlin, consist simply of a series in powers of $\dot{\gamma}$." (See Ref. 11, p. 395). Here we present a comparison of the two kinds of expansions for two flows: steady shear flow and steady elongational flow.

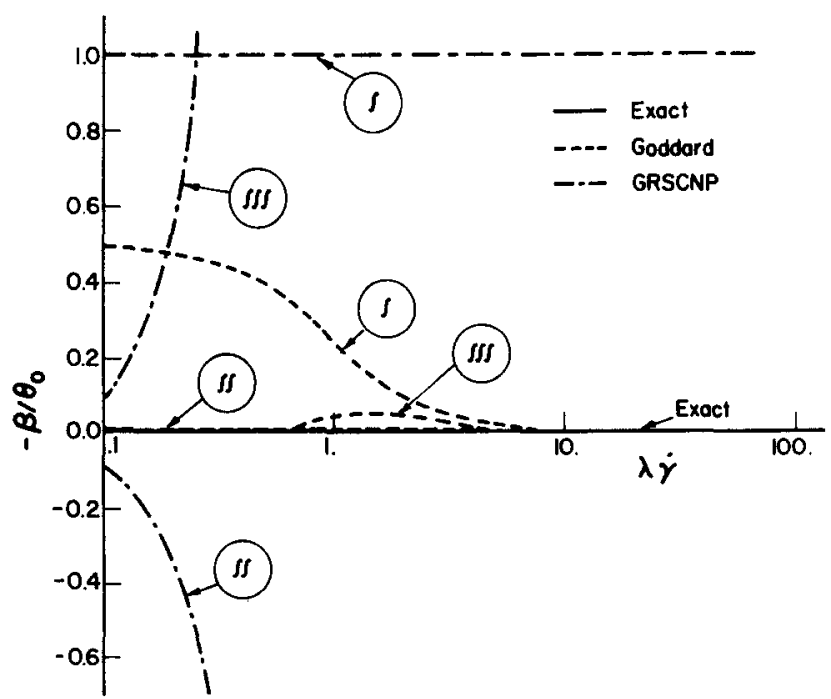

FIG. 3. Comparison similar to Fig. 1, for secondary normal stress function $\beta(\dot{\gamma})$. 

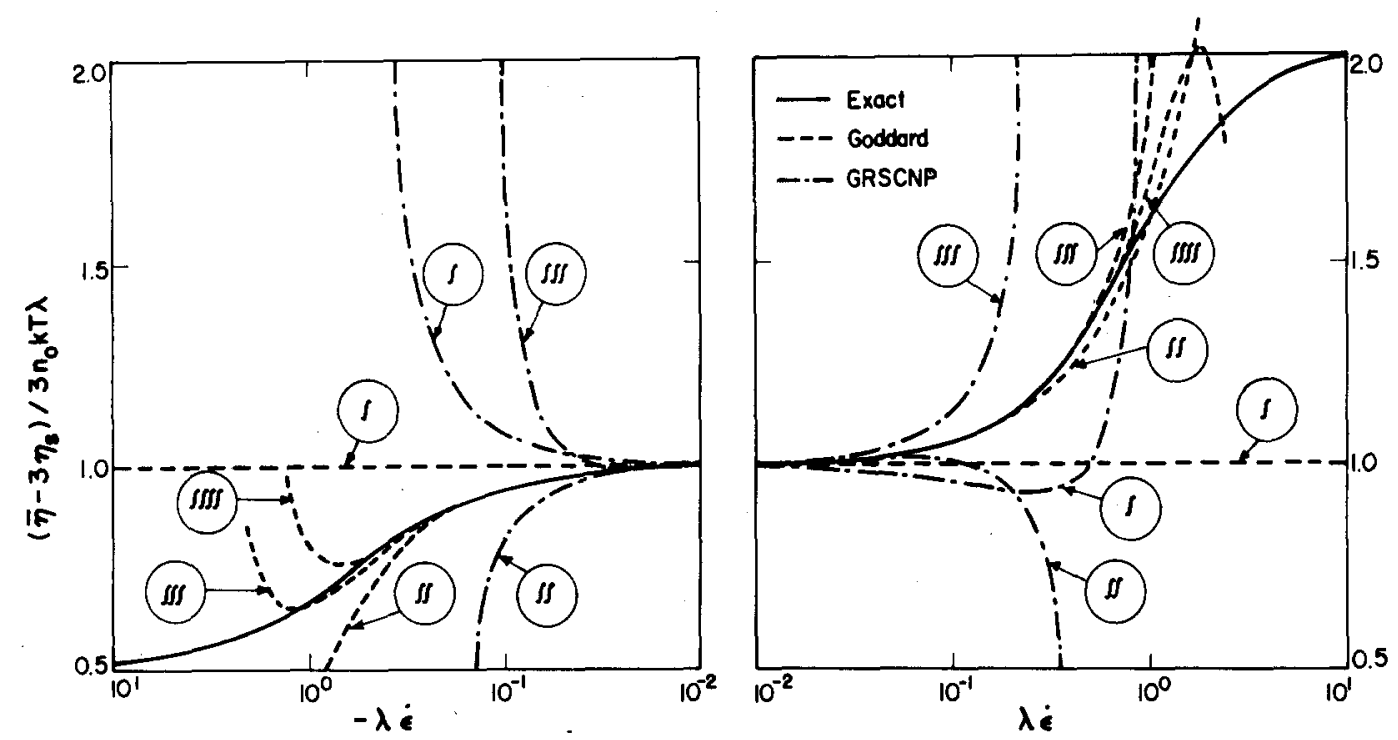

FIG. 4. Comparison of the exact elongational viscosity (as computed from the results of Bird, Johnson, and Stevenson ${ }^{19}$ ) for solutions of rigid rodlike macromolecules with the results obtained using a finite number of terms of the Goddard and the GRSCNP expansions.

\section{A. Steady shear flow}

For the steady shear flow $v_{x}=\dot{\gamma} y, v_{y}=v_{z}=0$, three material functions $\eta, \theta$, and $\beta$ may be measured; these functions of the shear rate $\dot{\gamma}$ are defined by

$$
\begin{aligned}
& \tau_{y x}=-\eta \dot{\gamma}, \\
& \tau_{x x}-\tau_{y y}=-\theta \dot{\gamma}^{2}, \\
& \tau_{y y}-\tau_{x x}=-\beta \dot{\gamma}^{2} .
\end{aligned}
$$

For the rigid macromolecule model defined in Sec. II, the functions $\eta(\dot{\gamma}), \theta(\dot{\gamma})$, and $\beta(\dot{\gamma})$ have been computed by Stewart and Sorensen. ${ }^{17}$ Their computed results have been shown in Figs. $1-3$ as the solid curves. On the same graphs are shown the curves which one obtains by using a total of one, two, or three terms in the memory integral expansions. The kine matic tensors needed for the two expansions are

$$
\dot{\Gamma}^{\prime}=\left(\begin{array}{ccc}
\dot{\gamma} \sin \dot{\gamma}\left(t-t^{\prime}\right) & \dot{\gamma} \cos \dot{\gamma}\left(t-t^{\prime}\right) & 0 \\
\dot{\gamma} \cos \dot{\gamma}\left(t-t^{\prime}\right) & -\dot{\gamma} \sin \dot{\gamma}\left(t-t^{\prime}\right) & 0 \\
0 & 0 & 0
\end{array}\right)
$$

TABLE III. Elongational viscosity for solutions of rigid macromolecules as obtained from the Goddard expansion [y $=\lambda \dot{\varepsilon}]$.

\begin{tabular}{ll}
\hline \hline $\begin{array}{l}\text { Contribution due } \\
\text { to term of order: }\end{array}$ & $\frac{\bar{\eta}-3 \eta_{s}}{3 n_{0} k T \lambda}$ \\
\hline 1 & 1 \\
2 & $\frac{3}{5} y$ \\
3 & $\frac{9}{35} y^{2}$ \\
4 & $-\frac{27}{175} y^{2}$ \\
\hline \hline
\end{tabular}

$$
\boldsymbol{\gamma}^{[1]}=\left(\begin{array}{ccc}
0 & \dot{\gamma} & 0 \\
\dot{\gamma} & -2 \dot{\gamma}^{2}\left(t-t^{\prime}\right) & 0 \\
0 & 0 & 0
\end{array}\right)
$$

Substitution of this $\dot{\Gamma}^{\prime}$ and $G_{I}, G_{I I}, \ldots$ into Eq. (12) give the results shown for the Goddard series; substitution of the above $\gamma^{[1]}$ and $G_{1}, G_{2}, \ldots$ from Eqs. (23)-(26) (Ref. 6) into Eq. (3) (Ref. 6) give the results shown for the GRSCNP series. It is clear that even the first few terms of the co-rotational Goddard expansion give rather good approximations to the exact curves over a wide range of shear rates. The GRSCNP expansion gives results which are only about as good as the KirkwoodPlock power series expansion [see Eq. (24), or curve $\mathrm{B}$ in Fig. 1 of Ref. 18; also Eq. (6.7) of Ref. 16].

The analytical results for the two memory-integral expansions are summarized in Tables I and II.

TABLE IV. Elongational viscosity for solutions of rigid macromolecules as obtained from the GRSCNP expansion $[y=\lambda \epsilon]$.

\begin{tabular}{ll}
\hline $\begin{array}{l}\text { Contribution } \\
\text { due to term. } \\
\text { of order: }\end{array}$ & $\frac{\bar{\eta}-3 \eta_{s}}{3 n_{0} k T \lambda}$ \\
\hline 1 & $\frac{2}{5}+\frac{3}{5(1+2 y)(1-y)}$ \\
2 & $\frac{56 y+64 y^{2}}{35(1+2 y)(1+4 y)}-\frac{14 y-8 y^{2}}{35(1-y)(1-2 y)}$ \\
3 & $\frac{y^{2}\left(6840+36528 y+60576 y^{2}+36864 y^{3}\right)}{35(1+2 y)(1+3 y)(1+4 y)(1+6 y)(10+12 y)}$ \\
& $+\frac{y^{2}\left(720-576 y+216 y^{2}\right)}{35(1-y)(1-2 y)(1-3 y)(10-6 y)}$ \\
& $+\frac{12 y^{3}(y+2)}{7(1-y)(1+2 y)(1+3 y)(10+3 y)}$ \\
\hline \hline
\end{tabular}




\section{B. Elongational flow}

For steady elongational flow $v_{z}=\stackrel{\circ}{\epsilon} z, v_{x}=-\frac{1}{2} \dot{\epsilon} x, v_{y}$ $=-\frac{1}{2} \dot{\epsilon}_{y}$, one material function, the "elongational viscosity" $\bar{\eta}$, may be defined:

$$
\tau_{x x}-\tau_{z z}=\bar{\eta} \dot{\epsilon}
$$

For the rigid macromolecular model under consideration, the function $\bar{\eta}(\dot{\epsilon})$ has been calculated by Bird, Johnson, and Stevenson. ${ }^{19}$ Their calculated results, extended to negative elongation rates, are shown in Fig. 4 as the solid curves. On the same graphs are shown the curves which are obtained by using one, two, or three terms in the two memory-integral expansions, with the following kinematic tensors:

$$
\begin{aligned}
& \dot{\Gamma}^{\prime}=\left(\begin{array}{ccc}
-1 & 0 & 0 \\
0 & -1 & 0 \\
0 & 0 & 2
\end{array}\right) \stackrel{\circ}{\epsilon} \\
& \gamma^{[1]}=\left(\begin{array}{ccc}
-\exp \dot{\epsilon}\left(t-t^{\prime}\right) & 0 & 0 \\
0 & -\exp \dot{\epsilon}\left(t-t^{\prime}\right) & 0 \\
0 & 0 & 2 \exp \left[-2 \dot{\epsilon}\left(t-t^{\prime}\right)\right]
\end{array}\right) \dot{\epsilon}
\end{aligned}
$$

The Goddard expansion is term by term identical to the power-series expansion in $\lambda \dot{\epsilon}$ [see Eq. (16.5) of Ref. 16]. Once again it is evident that the Goddard series is an improvement over the GRSCNP expansion. The analytical results corresponding to Fig. 4 are summarized in Tables III and IV.

\section{CONCLUSIONS}

(1) If one can solve the kinetic theory equations to obtain the stress tensor for an arbitrary time-dependent ir rotational flow, the constitutive equation for arbitrary flows can be written down at once [cf. Eq. (11)]. This suggests that more efforts should be directed towards the solution of kinetic-theory equations for time-dependent irrotational flows.

(2) The Goddard series is seen to be more useful than the GRSCNP series for presenting macromolecular kinetic theory results, since the former has better convergence properties.

(3) Since the kinetic theory model used here is known to exhibit qualitatively many of the properties of real fluids, the Goddard series should be more useful for applied hydrodynamic calculations than the GRSCNP series. ${ }^{20}$

Note added in proof: Although the modified Oldroyd expansion in Ref. 4 gives the viscosity and other steady- state material functions correctly through third order, the viscosity function is not realistic for all shear rates. Specifically one obtains

$$
\frac{[\eta]}{[\eta]_{0}}=\frac{1715-1792(\lambda \dot{\gamma})^{2}+128(\lambda \dot{\gamma})^{4}}{1715-910(\lambda \dot{\gamma})^{2}-880(\lambda \dot{\gamma})^{4}}
$$

which behaves improperly.

\section{ACKNOWLEDGMENTS}

The authors wish to acknowledge financial assistance provided by the Graduate School of the University of Wisconsin, the National Science Foundation (Grant GK24749), and the Vilas Trust Fund. Numerous conversations with Mr. Daniel J. Segalman and Professor M. W. Johnson, Jr., have been helpful.

${ }^{1}$ A. S. Lodge, Trans. Faraday Soc. 52, 120 (1956).

${ }^{2} \mathrm{H}$. Giesekus, Kolloid-Z. 147-149, 29 (1956); Errata in fn. 18 of Rheol. Acta 1, 404 (1961).

${ }^{3}$ S. Prager, Trans. Soc. Rheol. 1, 53 (1957).

${ }^{4}$ R. B. Bird, Zeitschrift für angewandte Mathematik und Physik 23, 157 (1972).

${ }^{5}$ R. B. Bird and R. C. Armstrong, J. Chem. Phys. 56, 3680 (1972).

${ }^{6}$ R. C. Armstrong and R. B. Bird, J. Chem. Phys. 58, 2715 (1973); Erratum: in Eq. (22), 27/280 should read 27/980.

${ }^{7}$ A. E. Green, R. S. Rivlin, and A. J. M. Spencer, Arch. Rat. Mech. Anal. 3, 82 (1959).

${ }^{8}$ A. E. Green and R. S. Rivlin, Arch. Rat. Mech. Anal. 1, I (1957); 4, 387 (1960).

${ }^{9}$ B. D. Coleman and W. Noll, Rev. Mod. Phys. 33, 239 (1961); Errata, ibid. 36, 1103 (1964).

${ }^{10}$ A. C. Pipkin, Rev. Mod. Phys. 36, 1034 (1964).

${ }^{11}$ J. D. Goddard, Trans. Soc. Rheol. 11, 381 (1967); Erratum, in Eq. (2.15) change $W^{\dagger}\{\tau\}$ to $W^{\dagger}\{t\}$.

${ }^{12}$ J. D. Goddard and C. Miller, Rheol. Acta 5, 177 (1966).

${ }^{13} \mathrm{~L}$. E. Malvern, Introduction to the Mechanics of a Continuous Medium (Prentice-Hall, Englewood Cliffs, NJ, 1969), Sec. 6.7.

${ }^{14}$ B. D. Coleman, H. Markovitz, and W. Noll, Viscometric Flows of Non-Newtonian Fluids (Springer-Verlag, New York, 1966), Sec. 6.

${ }^{15}$ J. G. Oldroyd, Proc. R. Soc. A 200, 523 (1950); 245, 278 (1958).

${ }^{16}$ R. B. Bird, H. R. Warner, Jr., and D. C. Evans, Fortschr. Hochpolymeren-Forschung 8, 1 (1971).

${ }^{17}$ W. E. Stewart and J. P. Sorensen, Trans. Soc. Rheol. 16, 1 (1972).

${ }^{18}$ J. G. Kirkwood and R. J. Plock, J. Chem. Phys. 24, 665 (1956).

${ }^{19}$ R. B. Bird, M. W. Johnson, Jr., and J. F. Stevenson, Proceedings of the 5th International Congress on Rheology (Tokyo University Press, Tokyo, 1970), Vol. 4, pp. 159-168.

${ }^{20}$ R. B. Bird, O. Hassager, and S. I. Abdel-Khalik, AIChE J. 20,000 (1974). 